\title{
Long noncoding TMPO antisense RNA 1 promotes hepatocellular carcinoma proliferation and epithelial-mesenchymal transition by targeting the microRNA-126-3p/LRP6/ $\beta$-catenin axis
}

\author{
Weifeng Huang ${ }^{1}$, Qingsong Chen ${ }^{2}$, Jiangweng Dai ${ }^{3}$, Yuke Zhang ${ }^{1}$, Yan Yi ${ }^{1}$, Xufu Wei ${ }^{1}$ \\ ${ }^{1}$ Department of Hepatobiliary Surgery, The First Affiliated Hospital of Chongqing Medical University, Chongqing, China; ${ }^{2}$ Department of Traumatology, \\ Chongqing University Central Hospital, Chongqing, China; ${ }^{3}$ Department of Oncology, Chengdu Fifth People's Hospital, Chengdu, China \\ Contributions: (I) Conception and design: W Huang; (II) Administrative support: X Wei; (III) Provision of study materials or patients: X Wei; (IV) \\ Collection and assembly of data: W Huang, Q Chen, J Dai; (V) Data analysis and interpretation: W Huang, Q Chen, J Dai, Y Zhang, Y Yi; (VI) \\ Manuscript writing: All authors; (VII) Final approval of manuscript: All authors. \\ Correspondence to: Xufu Wei. Department of Hepatobiliary Surgery, The First Affiliated Hospital of Chongqing Medical University, Youyi Road, \\ Yuanjiagang, Yuzhong District, Chongqing 400016, China. Email: xwei@hospital.cqmu.edu.cn.
}

\begin{abstract}
Background: Long noncoding RNAs (lncRNAs) play a central role in the pathogenesis of various tumors, including hepatocellular carcinoma (HCC). TMPO antisense RNA 1 (TMPO-AS1) has been reported in many tumors. Nevertheless, the underlying mechanism whereby TMPO-AS1 influences HCC remains unclear. Our research aimed to reveal the molecular mechanism governing the function of TMPO-AS1 in HCC.

Methods: TMPO-AS1 expression levels in HCC tissues/cells were evaluated using reverse transcriptasepolymerase chain reaction. The effect of TMPO-AS1 on the progression of HCC was observed by Cell Counting Kit-8 (CCK8), clone formation, wound healing, and transwell. The direct interaction between TMPO-AS1 and microRNA (miR)-126-3p was observed using a dual-luciferase reporter.

Results: We found TMPO-AS1 expression to be remarkably higher in HCC specimens and associated with poor prognosis. Silencing of TMPO-AS1 not only inhibited HCC cell proliferation but also significantly reduced epithelial-to-mesenchymal transition-induced invasion and migration to a remarkable degree. According to the results from the online database analysis tools implemented to identify if TMPO-AS1 could target miR-126-3p, we found that miR-126-3p had a negative relationship with TMPO-AS1 in HCC specimens. Meanwhile, the luciferase reporter assay confirmed that TMPO-AS1 could directly act on miR126-3p. Moreover, the silencing of miR-126-3p dramatically abolish the inhibitive influence of sh-TMPOAS1 on HCC development.

Conclusions: Our research demonstrated that TMPO-AS1 acts as a sponge for the tumor suppressor miR-126-3p in HCC and promotes the expression of LRP6 indirectly. Taken together, our results show that TMPO-AS1 may be regarded as a novel therapeutic target in the treatment of liver cancer.
\end{abstract}

Keywords: TMPO antisense RNA 1 (TMPO-AS1); miR-126-3P; epithelial-mesenchymal transition (EMT); proliferation; invasion

Submitted Sep 22, 2021. Accepted for publication Nov 04, 2021.

doi: 10.21037/atm-21-5593

View this article at: https://dx.doi.org/10.21037/atm-21-5593

\section{Introduction}

Liver cancer is one of the most commonly diagnosed cancers and a leading cause of global cancer-related mortalities. Hepatocellular carcinoma (HCC) is the most common primary liver cancer, accounting for about 75$85 \%$ (1). Although there are several treatments available for HCC, it continues to have a poor prognosis with a high recurrence rate after traditional therapies $(2,3)$. Recent 
studies report that long noncoding RNA (lncRNA) may function as a novel prognostic marker and therapeutic target for HCC (4).

Long noncoding RNAs belong to a group of noncoding RNA molecules containing more than 200 nucleotides (5) and were once thought to be transcriptional 'garbage'. However, emerging evidence has demonstrated that lncRNAs are not genomic 'noise' but rather functional RNA molecules, which participate in critical cellular processes such as growth and differentiation as well as the pathogenesis of diseases, including cancer (6). Thus, lncRNAs have become an increasingly popular focus of cancer research. A large body of emerging studies has proven that IncRNAs exhibit aberrant expression in HCC and extensively participate in cancer pathogenesis by regulating cell anti-tumor drug resistance, growth, apoptosis, and epithelial-to-mesenchymal transition (EMT) and invasion (7-10). The TMPO antisense RNA 1 (TMPO-AS1) gene has been proposed to have a carcinogenic role in various cancers. In cervical cancer, TMPO-AS1 is positively correlated with an aggressive phenotype and a poor prognosis (11). Moreover, recent studies have shown TMPOAS1 overexpression stimulates cell division and migration in prostate cancer, thus serving as a biomarker for disease diagnosis and prognosis (12). Also, TMPO-AS1 has been shown to promote gastric cancer cells migration and invasion by activating the expression of EMT modulators (13). However, the underlying role of TMPO-AS1 in HCC development remains elusive.

MicroRNAs (miRNAs) are small noncoding, endogenously expressed RNAs comprised of 19-25 nucleotides, which regulate cellular biological processes in combination with messenger RNAs (mRNAs) $(14,15)$. At present, a few studies have reported on a type of competitive endogenous RNA (ceRNA), also known as microRNA sponge, which indirectly regulates the expression of mRNA through competitive binding of miRNAs $(16,17)$. Many lncRNAs can act as miRNA sponges to regulate tumor progression (18). However, the capacity of TMPO-AS1 to regulate proliferation, migration, and EMT of HCC, and if this regulation is derived from sponging a particular miRNA, remains to be elucidated.

This study aimed to investigate the biological function and molecular mechanism of TMPO-AS1 in HCC, to verify the expression profile of TMPO-AS1 in HCC tissues and cell lines and to explore the relationships between TMPO-AS1, miR-126-3p, LRP6, and $\beta$-catenin signaling pathway in HCC. Results from this study may lead to a new understanding of HCC occurrence and development and may provide a new therapeutic option for the treatment of HCC. We present the following article in accordance with the MDAR reporting checklist (available at https://dx.doi. org/10.21037/atm-21-5593).

\section{Methods}

\section{Patients and tissue specimens}

Tissue specimens were obtained from 53 Chinese HCC patients undergoing hepatectomy for primary tumors at the Department of Hepatobiliary Surgery of The First Affiliated Hospital of Chongqing Medical University between March 2018 and July 2020. The basic characteristics of all patients are outlined in Table 1. All procedures performed in this study involving human participants were in accordance with the Declaration of Helsinki (as revised in 2013). The study was approved by the Medical Ethics Committee of the First Affiliated Hospital of Chongqing Medical University (No. 2021-156) and informed consent was taken from all the patients.

\section{Cell culture}

The human HCC cell lines Hep3B, Huh7, SMMC-7721, Bel-7402, SK-Hep-1, LM9, and human normal liver cell line L-02 were procured from the Shanghai Institute of Biological Sciences, Chinese Academy of Sciences, and were subsequently cultured in Dulbecco Modified Eagle Medium (DMEM, Gibco, Amarillo, TX, USA) supplemented with $10 \%$ fetal bovine serum (FBS; Gibco, Amarillo, TX, USA; Life Technologies, Carlsbad, CA, USA) and 1\% penicillin/streptomycin (Hyclone, Logan, UT, USA) in an incubation chamber with $5 \% \mathrm{CO}_{2}$ at a constant temperature of $37^{\circ} \mathrm{C}$.

\section{Transfection}

Short hairpin RNAs (shRNAs) against TMPO-AS1 (shTMPO-AS1) were purchased from HanBio (Shanghai, China) along with the short hairpin negative control (sh-NC). The miR-126-3p inhibitor was purchased from GenePharma (Shanghai, China). Lipofectamine 2000 reagent (Invitrogen, Carlsbad, CA, USA) was employed for cell transfection following the protocols available on the manufacturer's website. Quantitative reverse transcription polymerase chain reaction (qRT-PCR) analysis was then used to monitor and quantify transfection efficiency after $48 \mathrm{~h}$. 
Table 1 Sociodemographic and medical characteristics of patients

\begin{tabular}{|c|c|c|}
\hline Clinicopathological characteristics & \multicolumn{2}{|c|}{ Number of cases } \\
\hline \multicolumn{3}{|l|}{ Age (years) } \\
\hline$<50$ & $21(63.6 \%)$ & $9(45 \%)$ \\
\hline$\geq 50$ & $12(36.4 \%)$ & $11(55 \%)$ \\
\hline Male & $26(78.8 \%)$ & $17(85 \%)$ \\
\hline Female & $7(21.2 \%)$ & $3(15 \%)$ \\
\hline \multicolumn{3}{|l|}{ Mass size $(\mathrm{cm})$} \\
\hline$\leq 5$ & $13(39.4 \%)$ & $18(90 \%)$ \\
\hline Absence & $23(69.7 \%)$ & $18(90 \%)$ \\
\hline Presence & $10(30.3 \%)$ & $2(10 \%)$ \\
\hline \multicolumn{3}{|l|}{ Histological differentiation } \\
\hline Poor & $3(9 \%)$ & $1(5 \%)$ \\
\hline Moderate & $25(75.8 \%)$ & $17(70 \%)$ \\
\hline Well & $5(15.2 \%)$ & $2(25 \%)$ \\
\hline \multicolumn{3}{|l|}{ Serum alpha-fetoprotein $(\mu \mathrm{g} / \mathrm{mL})$} \\
\hline$>400$ & $20(60.6 \%)$ & $12(60 \%)$ \\
\hline Absence & $5(15.2 \%)$ & $5(25 \%)$ \\
\hline Presence & $28(84.8 \%)$ & $15(75 \%)$ \\
\hline
\end{tabular}

\section{Quantitative real-time polymerase chain reaction (qRT-PCR) assay}

Total cellular RNA was isolated from HCC cells via TRIzol reagent (Invitrogen, Carlsbad, CA, USA) according to the manufacturer's guidelines. Reverse transcription of TMPOAS1 into single-stranded complementary DNA (cDNA) was conducted using a reverse transcription system kit (Takara, Shiga, Japan). Hairpin-it miRNA real-time quantitative reverse transcription polymerase chain reaction (qRTPCR) kit (GenePharm, Sunnyvale, CA, USA) was utilized for the reverse transcription of miR-126-3p. Then, qRTPCR assays were conducted with $2 \times$ SYBR Green qPCR Master Mix (Bimake, Houston, TX, USA) on the BioRad CFX96 Real-Time PCR Detection machine (Bio-Rad Laboratories, Hercules, CA, USA). U6 and glyceraldehyde 3-phosphate dehydrogenase (GAPDH) functioned as internal references for miR-126-3p and TMPO-AS1, respectively. The gene-specific primers were as follows: GAPDH, 5'-CTTTGGTATCGTGGAAGGACTC-3' (F), and 5'-GTAGAGGCAGGGATGATGTTCT-3' (R); TMPO-AS1, 5'-GACCTCTACAATCGGGCACTT-3' 
(F), and 5'-AGGATTCTTGCGGGTGGTG-3' (R); U6, 5'-CAGCACATATACTAAAATTGGAACG-3' (F), and 5'-ACGAATTTGCGTGTCATCC-3' (R); miR126-3p: 5'-CGCGTCGTACCGTGAGTAAT-3' (F) and 5'-AGTGCAGGGTCCGAGGTATT-3' (R). All primers were synthesized by Takara (Shiga, Japan). The comparative $2^{-\Delta \Delta C t}$ method was then implemented to analyze relative gene expression.

\section{Cell counting kit-8 (CCK-8) assay}

To assess cell viability, a CCK8 assay was performed according to the instructions of the manufacturer (Dojindo, Japan). After being transfected with sh-TMPO-AS1 and sh-NC for $24 \mathrm{~h}$, cells were reseeded into 96-well plates $\left(2 \times 10^{3}\right.$ cells/well $)$ and cultured for several days. Each well was replaced with a solution of $100 \mu \mathrm{L}$ of complete medium containing $10 \mu \mathrm{L}$ CCK8. Multiskan Spectrum (Thermo Fisher, Rockford, IL, USA) was employed to measure absorbance at $450 \mathrm{~nm}$.

\section{Colony formation assay}

Cells that had been transfected for 24 hours were reseeded onto $6 \mathrm{~cm}$ plates at a density of 500 cells/plate and were subsequently shaken well and cultured for 2 weeks, such that individual cells grew into a mass of cells visible to the naked eye. Next, the plates were fixed followed by staining with $3.7 \%$ methanol with $0.1 \%$ crystal violet.

\section{Western blot assay}

After $72 \mathrm{~h}$ of transfection, the total cellular protein was separated using sodium dodecyl sulfate polyacrylamide gel electrophoresis (SDS-PAGE) and transferred into polyvinylidene fluoride membranes. Membranes were incubated in QuickBlock ${ }^{\mathrm{TM}}$ blocking buffer (Beyotime, Shanghai, China) for $15 \mathrm{~min}$, then washed with Tris Buffered Saline with Tween (TBST, Solarbio, Beijing, China) buffer solution (20 mM Tris- $\mathrm{HCl}, 137 \mathrm{mM} \mathrm{NaCl}$, and $0.1 \%$ Tween 20 ). The protein bands were then incubated overnight with the relevant primary antibodies at $4{ }^{\circ} \mathrm{C}$. The primary antibodies utilized include anti-LRP6 (1:1,000, Abways, Shanghai, China), anti- $\beta$-catenin (1:1,000, Cell Signaling Technology, Danvers, MA, USA), anti-Ecadherin (1:2,000, Affinity, Piscataway, NJ, USA), anti-Ncadherin (1:1,000, Cell Signaling Technology, Danvers, MA, USA), anti-vimentin (1:1,000, Bimake, Houston, TX, USA),
anti-Snail (1:1,000, Cell Signaling Technology, Danvers, MA, USA), and anti-GAPDH (1:20,000, Proteintech, Wuhan, China). The next day, the blots were washed with a mixture of TBS and Tween 20. The membranes were then exposed to secondary antibodies at room temperature for 1 hour. Next, the membranes were washed with TBST three times ( $5 \mathrm{~min}$ each). The protein bands were analyzed with an enhanced chemiluminescence (ECL) kit (Merck Millipore, Ltd., Darmstadt, Germany) and a Vilber Fusion FX7 (Fusion, Paris, France). Protein expression was quantified with ImageJ software (National Institutes of Health, Bethesda, MD, USA).

\section{Wound healing assay}

The transfected HCC cells were seeded into six-well plates. Afterward, cell scratches were created with $10-\mu \mathrm{L}$ sterile pipette tip. Representative images were captured under a bright inverted microscope (Olympus, Tokyo, Japan). Then, the wound infliction area was measured using the Image J system (National Institutes of Health, Bethesda, USA).

\section{Transwell assay}

To assess cell migratory or invasive ability, transwell inserts (Costar, Cambridge, MA, USA) were used either uncoated or coated with Matrigel (BD Biosciences, Franklin Lakes, NJ, USA), respectively. After $24 \mathrm{~h}$ of transfection, cells were incubated in the upper chamber with serum-free medium. At the same time, DMEM (Gibco, Amarillo, TX, USA) containing $10 \%$ FBS was placed in the lower chamber. The cells were incubated for $24 \mathrm{~h}$ at $37^{\circ} \mathrm{C}$, and noninvaded cells were carefully eliminated via cotton swabs. Meanwhile, the invaded cells located on the membrane's underside were fixed with $95 \%$ methanol and stained with crystal violet for $30 \mathrm{~min}$ at room temperature. Five randomized images were captured using the Carl ZEISS microscope system (ZEISS, Jena, Germany). Finally, the stained cells were counted and averaged across five random fields.

\section{Dual-luciferase reporter assay}

Dual-luciferase reporter assay was implemented to confirm the interaction between TMPO-AS1 and miR-126-3p. Wild-type (Wt) and mutant (Mut) miR-126-3p 3'-UTR were inserted into the pSI-Check2 vector (HanBio, New Taipei City 221, Taiwan). Then, sh-NC or sh-TMPOAS1 was cotransfected with Wt miR-126-3p 3'-UTR or 
Mut miR-126-3p 3'-UTR into Huh7 cells. After 48 hours, luciferase activity was measured with the dual-luciferase reporter system (Promega, Madison, WI, USA).

\section{Bioinformatics analysis}

A Kaplan-Meier plotter (https://www.kmplot.com/ analysis/) was employed to detect the relationship between TMPO-AS1 level and overall patient survival quality. The possible target gene of TMPO-AS1 was predicted using starBase Version 3.0 (http://starbase.sysu.edu.cn/). The difference in the expression of TMPO-AS1 between normal and tumor liver tissues was analyzed using the online Atlas of Noncoding RNAs in Cancer (TANRIC) database (https://ibl.mdanderson.org/tanric/_design/basic/ main.html).

\section{Statistical analyses}

GraphPad Prism (version 8.0, GraphPad, San Diego, CA, USA) was used for statistical analysis. Data are presented as mean \pm standard deviation (SD). The normality of quantitative data was also examined. The student's $t$-test was used to analyze differences between two groups of the normally distributed data. One-way analysis of variance was employed to compare statistical differences among three or more groups using the Tukey-Kramer test. The differences between paracarcinoma and HCC tissues were examined using Wilcoxon matched-pairs signed rank test. $\mathrm{P}<0.05$ was considered as indicating statistical significance.

\section{Results}

\section{TMPO-AS1 is overexpressed and associated with poor prognosis in $\mathrm{HCC}$}

A qRT-PCR analysis conducted to measure TMPOAS1 levels revealed that TMPO-AS1 was remarkably overexpressed in HCC tissues compared to pericarcinomatous tissues (Figure 1A). It exhibited elevated levels in HCC lines when compared to healthy liver cells (L-O2). Of all HCC cell lines, TMPO-AS1 expression was greatest in Hep3B and Huh7 (Figure 1B). Additionally, TANRIC database results consistently indicated higher TMPO-AS1 expression in HCC tissues than in normal liver tissues (Figure 1C). As shown in Figure 1D, patients with lower levels of TMPOAS1 expression had a distinctly better median overall survival time than individuals that exhibited higher TMPO-
AS1 expression levels (59.7 vs. 46.57 months, respectively).

\section{Decreased TMPO-AS1 expression blocks proliferation in HCC cells}

To investigate the effect of TMPO-AS1 on the biological function of HCC cells, we transfected Hep3B and Huh7 cells with sh-TMPO-AS1\#1/2/3 and found inhibition efficiency was caused by sh-RNA\#2 (Figure $2 A$ ). We therefore selected sh-TMPO-AS1\#2 to silence TMPOAS1 in subsequent experiments. Firstly, CCK8 assay results indicated that knockdown of TMPO-AS1 remarkably reduced the proliferative capacity of Hep3B and Huh7 cells (Figure 2B), and a colony formation assay showed that TMPO-AS1 silencing prominently suppresses HCC cell growth compared to sh-NC populations (Figure 2C). These findings suggest that TMPO-AS1 regulates the progression of HCC through the regulation of HCC cell proliferation.

\section{Knockdown of TMPO-AS1 represses HCC cell migration and invasion in vitro}

As shown earlier, elevated TMPO-AS1 levels were observed in HCC patients with advanced-stage disease (i.e., higher tumor-node-metastasis stages) and portal vein tumor thrombosis. Therefore, we speculated TMPO-AS1 might be crucial in the metastasis of liver cancer cells. To verify this conjecture, two experimental schemes were adopted to explore differences in cell migration and invasion following TMPO-AS1 downregulation. First, we performed a wound healing assay to analyze HCC cells' motility in $24 \mathrm{~h}$ and found silencing of TMPO-AS1 repressed cell migration in Hep3B (Figure 3A) and Huh7 (Figure 3B) cells. Consistent with these results, transwell assays indicated that the downregulation of TMPO-AS1 minimized the quantity of migrated and invaded cells compared with shNC (Figure 3C,3D). Since EMT is critical for the enabling the metastasis of cancer cells, we further explored whether TMPO-AS1 regulates the induction of EMT-related proteins in HCC cells. Western blot analysis illustrated that TMPO-AS1 silencing intensified the epithelial protein expression but repressed the expression levels of mesenchymal proteins, such as $\mathrm{N}$-cadherin, Vimentin, and Snail (Figure 3E). Taken together, these findings suggest TMPO-AS1 contributes to HCC metastasis by promoting cell migration and invasion. 
A

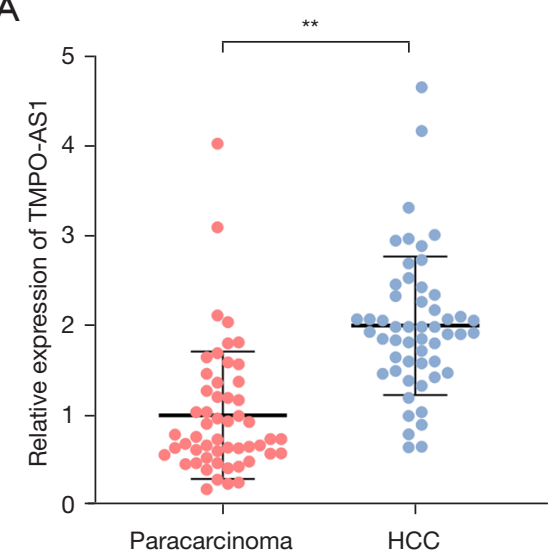

C

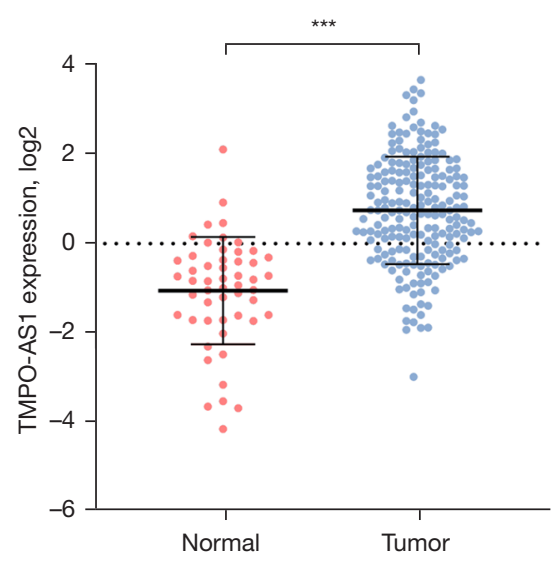

B

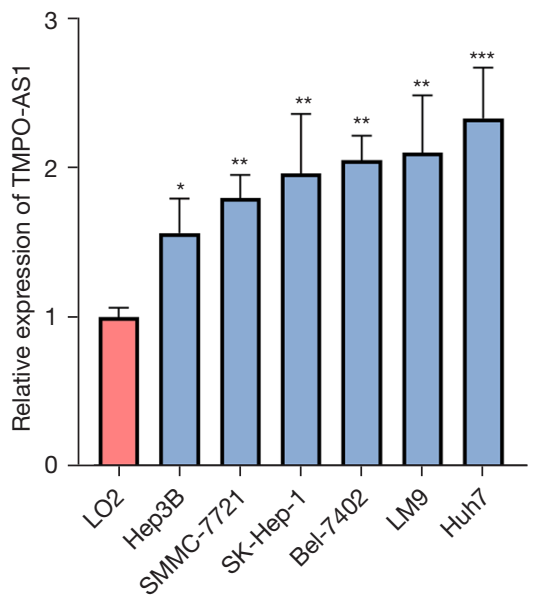

D

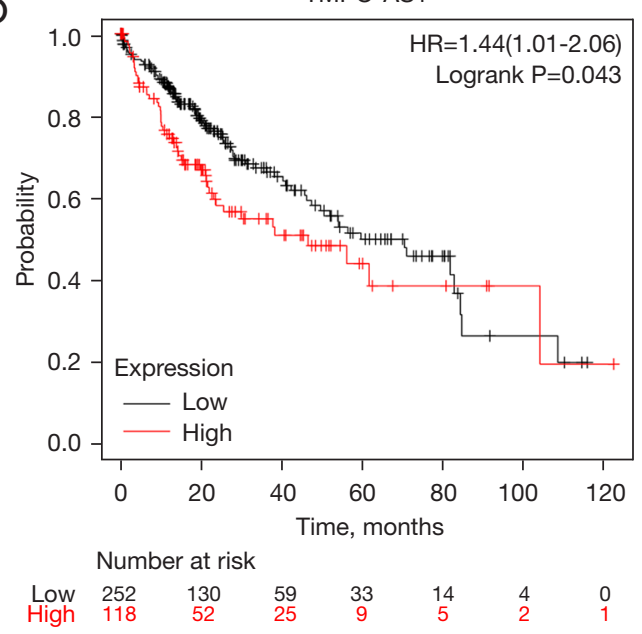

Median survival

\begin{tabular}{|c|c|}
\hline Low expression cohort (months) & High expression cohort (months) \\
\hline 59.7 & 46.57 \\
\hline
\end{tabular}

Figure 1 TMPO antisense RNA 1 (TMPO-AS1) expression in HCC tissues and cell lines. (A) Real-time quantitative reverse transcription polymerase chain reaction (qRT-PCR) was performed to evaluate the expression of TMPO-AS1 in 53 paired hepatocellular carcinoma (HCC) tissues. (B) TMPO-AS1 levels in control L-O2 cells and HCC cell lines were measuring using qRT-PCR. (C) Patient information was obtained from The Cancer Genome Atlas database to analyze TMPO-AS1 expression in the HCC tumor group ( $\mathrm{n}=200)$ compared with the normal control group $(\mathrm{n}=50)$. (D) The survival percentage was lower in HCC patients with higher TMPO-AS1 expression levels than in those with lower TMPO-AS1 expression levels. *, $\mathrm{P}<0.05$; **, $\mathrm{P}<0.01$; ***, $\mathrm{P}<0.001$.

\section{TMPO-AS1 promotes HCC progression by competitively binding to miR-126-3p and activating the LRP6/B-catenin axis}

To investigate the underlying molecular mechanisms by which TMPO-AS1 promotes HCC cell progression, we used the starBase (https://starbase.sysu.edu.cn/) 3.0 online database to predict the potential target genes of TMPOAS1. MiR-126-3p was identified as prospective loci TMPOAS1 may bind with. As illustrated in Figure 4A, TMPO-AS1 formed complementary base pairs with miR-126-3p. To 
A

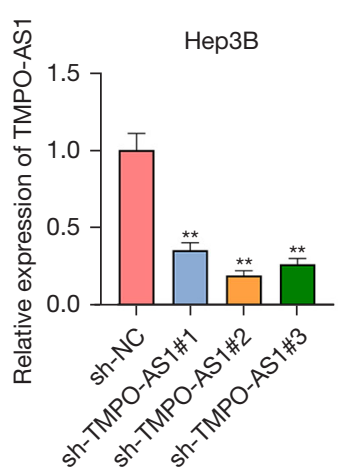

C

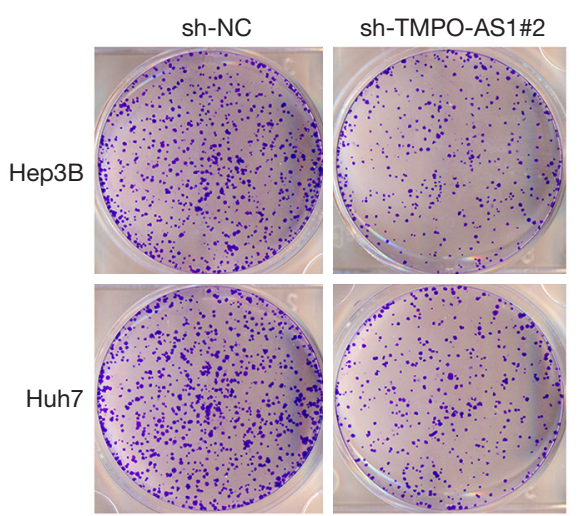

B
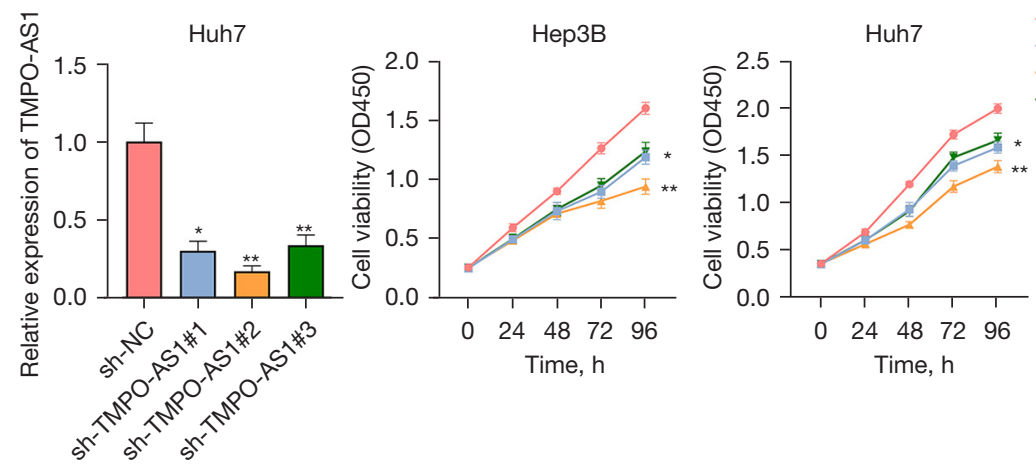

$\rightarrow$ sh-NC

- sh-TMPO-AS1\#1

- sh-TMPO-AS1\#2

* sh-TMPO-AS1\#3

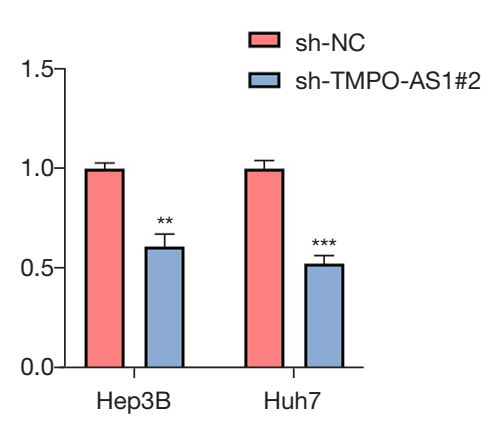

Figure 2 TMPO antisense RNA 1 (TMPO-AS1) regulates hepatocellular carcinoma (HCC) cell proliferation. (A) Real-time quantitative reverse transcription polymerase chain reaction analysis of TMPO-AS1 induction in Hep3B and Huh7 cells following transfected with three different types of sh-TMPO-AS1. (B) Cell Counting Kit-8 assays revealed TMPO-AS1 knockdown inhibits Hep3B and Huh7 cell proliferation. (C) Downregulation of TMPO-AS1 weakens the colony formation ability of $\mathrm{HCC}$ cells. ${ }^{*}, \mathrm{P}<0.05 ;{ }^{* *}, \mathrm{P}<0.01 ;{ }^{* * *}, \mathrm{P}<0.001$.

evaluate whether TMPO-AS1 directly interacts with miR126-3p, transfected Huh7 cells were analyzed using a dualluciferase gene reporter assay. As highlighted by Figure 4B, the luciferase activity was dramatically reduced following co-transfection with sh-TMPO-AS1 and Wt-miR-126-3p. However, the luciferase activity remained unchanged in the cells co-transfected with sh-TMPO-AS1 and Mut-miR126-3p.

To further assess the correlation between TMPOAS1 and miR-126-3p, the expression level of miR-126$3 \mathrm{p}$ in 53 paired HCC tissues was evaluated through qRTPCR analysis (Figure 4C). The induction of miR-126$3 \mathrm{p}$ was considerably lower in HCC tissues than in their paired normal tissues. Interestingly, Pearson's correlation analysis revealed an inverse relationship between TMPOAS1 and miR-126-3p (Figure 4D). Since previous research has reported that LRP6 is the downstream target of miR126-3p (19), we speculated that LRP6 and subsequent signaling pathways might be modulated by TMPO-AS1. As hypothesized, downregulation of TMPO-AS1 decreased the expression levels of LRP6 and beta-catenin in Hep3B and Huh7 cells (Figure 4E). Moreover, a miR-126-3p inhibitor was transfected into Hep3B and Huh7 cells (Figure $4 F$ ) to ascertain if miR-126-3p plays an intermediary role between TMPO-AS1 and LRP6 in liver cancer cells. Because Huh7 cells were observed to have a higher degree of transfection efficiency than Hep3B cells, Huh7 cells were used for successive experiments. Western blot analysis revealed that co-transfection with sh-TMPO-AS1 and the miR-126$3 p$ inhibitor restored the expression of LRP6 and betacatenin, which were downregulated by sh-TMPO-AS1 (Figure $4 G$ ). These findings prove TMPO-AS1 can interact with miR-126-3p to control LRP6 induction. In summary, we provided evidence that TMPO-AS1 could act as an endogenous 'sponge' by combining with miR-126-3p to counteract the miRNA-induced repression of LRP6. 

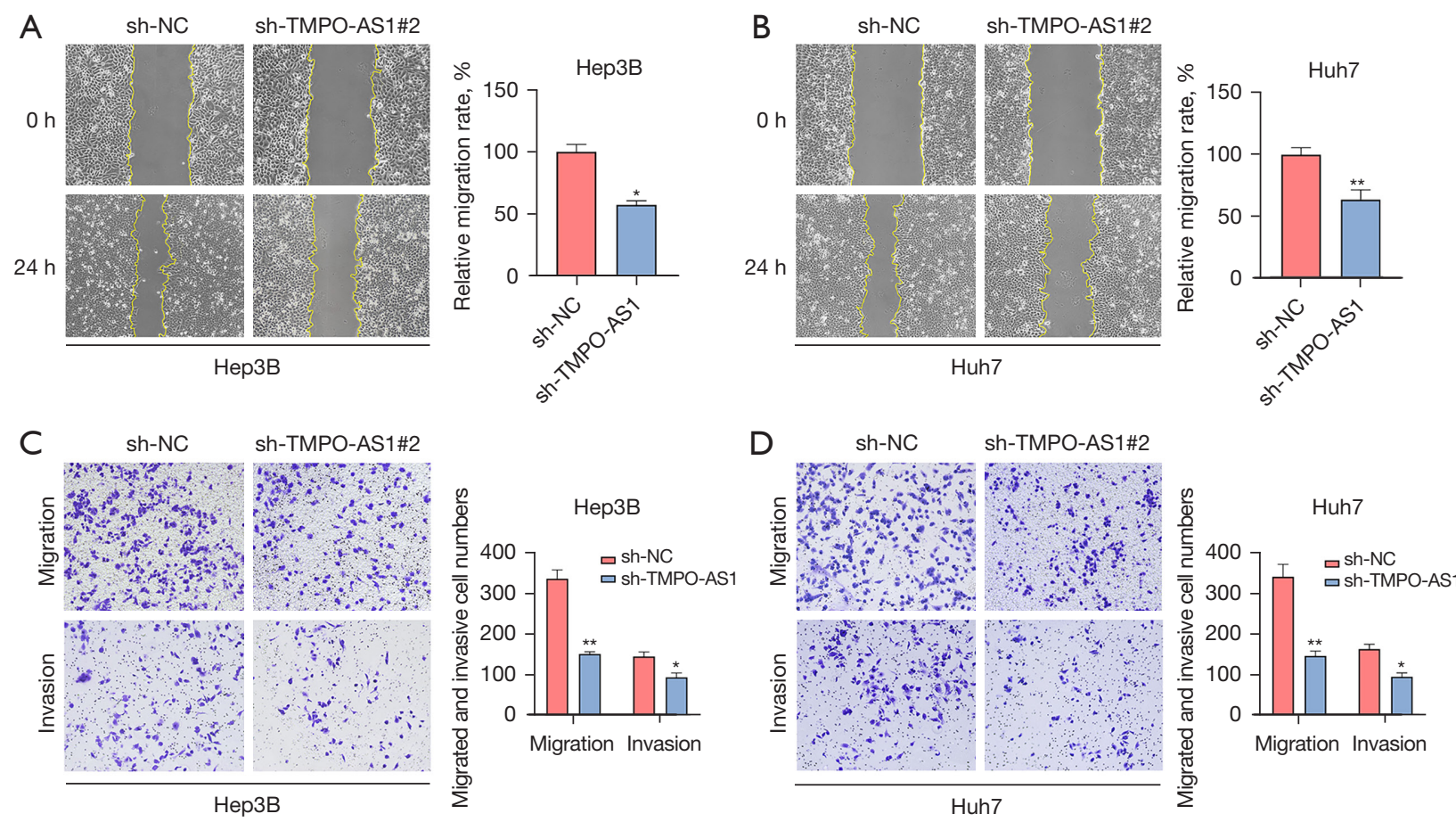

E

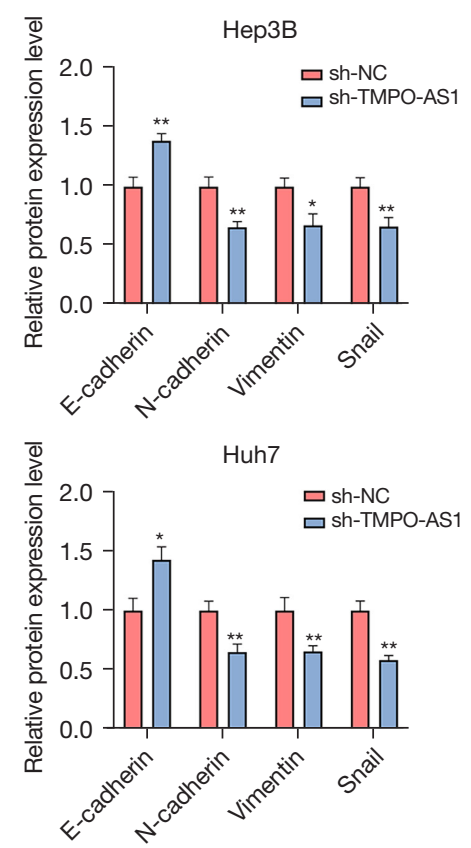

Figure 3 TMPO antisense RNA 1 (TMPO-AS1) promotes hepatocellular carcinoma (HCC) cell migration and invasion. (A,B) Wound healing analysis found that TMPO-AS1 is positively correlated with Hep3B and Huh7 cell metastasis. (C,D) Transwell assays revealed that TMPO-AS1 knockdown reduced the metastasis and invasion ability of Hep3B and Huh7 cells. Cells were stained with crystal violet. Magnification power 200x. (E) Western blot analysis was conducted to assess the expression level of epithelial-to-mesenchymal transition (EMT)-associated proteins, including E-cadherin, N-cadherin, vimentin, and Snail, in HCC cells transfected with sh-TMPO-AS1\#2. *, $\mathrm{P}<0.05 ;{ }^{* *}, \mathrm{P}<0.01$. 
A

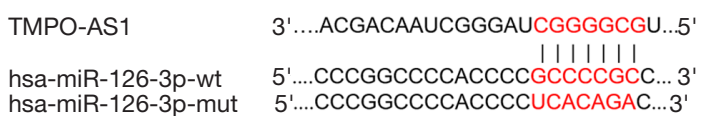

B

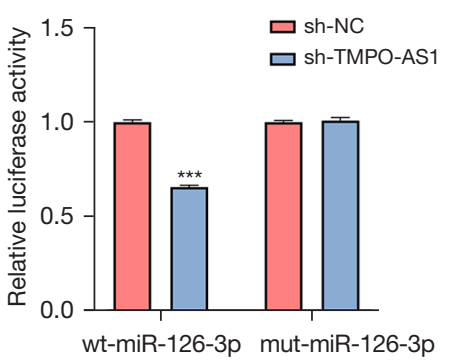

C

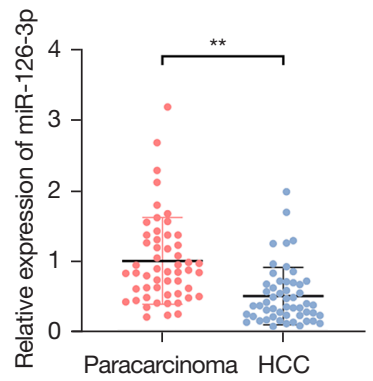

D

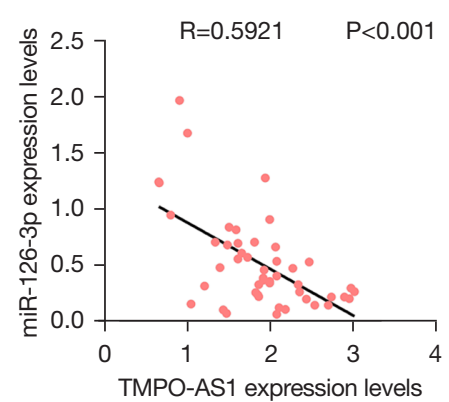

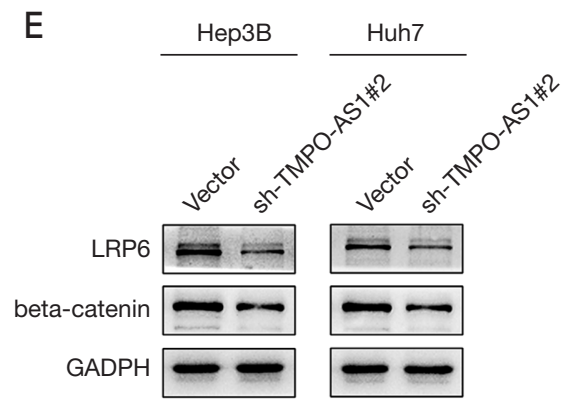
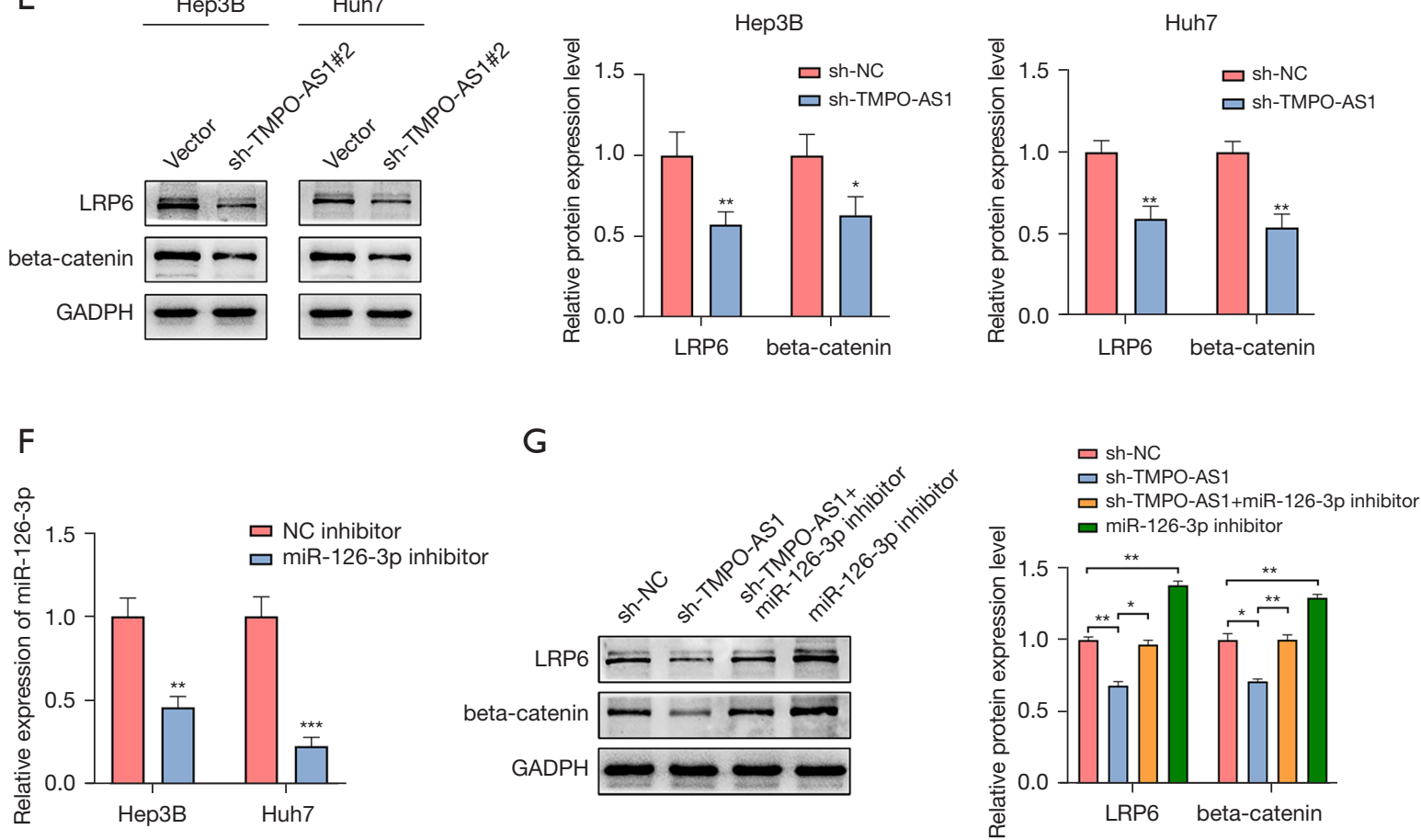

Figure 4 TMPO antisense RNA 1 (TMPO-AS1) activates the LRP6/ß-catenin signaling pathway by sponging miR-126-3p. (A) Wild or mutant type of miR-126-3p complementary binding sites for TMPO-AS1 were identified. (B) The luciferase reporter assay was employed to verify direct binding between TMPO-AS1 and miR-126-3p. (C) Real-time quantitative reverse transcription polymerase chain reaction (qRT-PCR) was utilized to analyze miR-126-3p expression in 53 paired HCC tissues. (D) Pearson's correlation analysis found a negative correlation between the expression profiles of TMPO-AS1 and miR-126-3p. (E) Western blotting was used to assess LRP6 and $\beta$-catenin expressions. (F) MiR-126-3p expression after transfecting Hep3B and Huh7 cells with a miR-126-3p inhibitor was analyzed using qRTPCR. (G) Western blot analyses showed that miR-126-3p inhibition reversed the changes in LRP6 and $\beta$-catenin expression levels. *, $\mathrm{P}<0.05 ;{ }^{* *}, \mathrm{P}<0.01 ; * * *, \mathrm{P}<0.001$. 


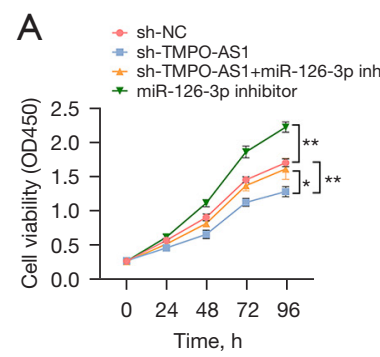

C
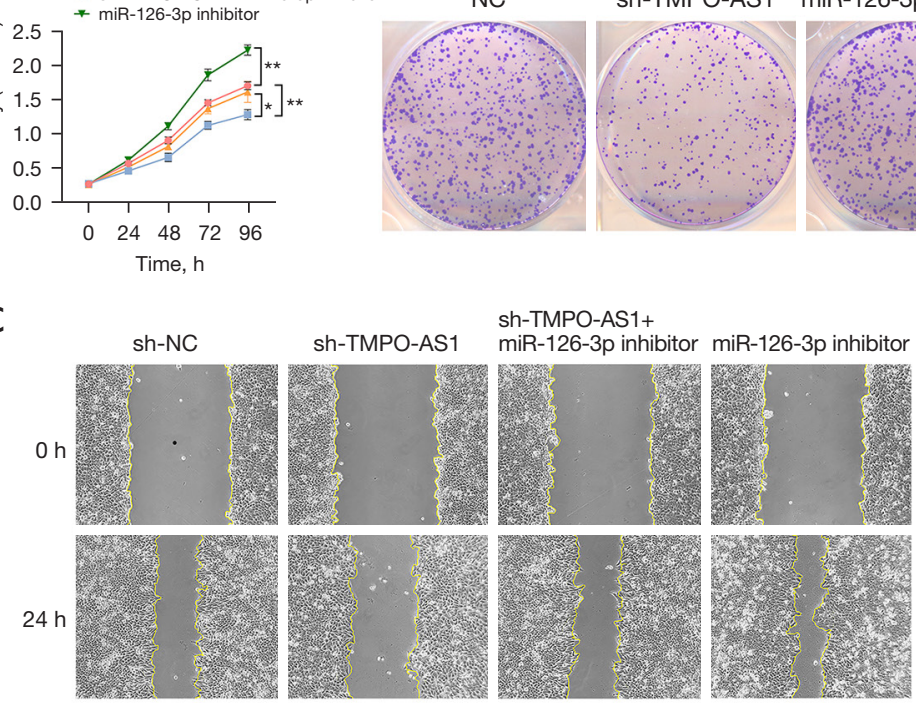

D

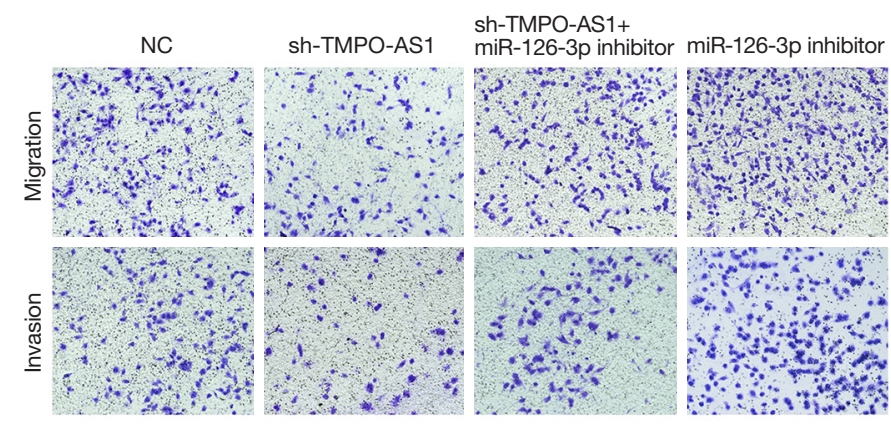

sh-TMPO-AS1+

sh-TMPO-AS1 miR-126-3p inhibitor miR-126-3p inhibitor
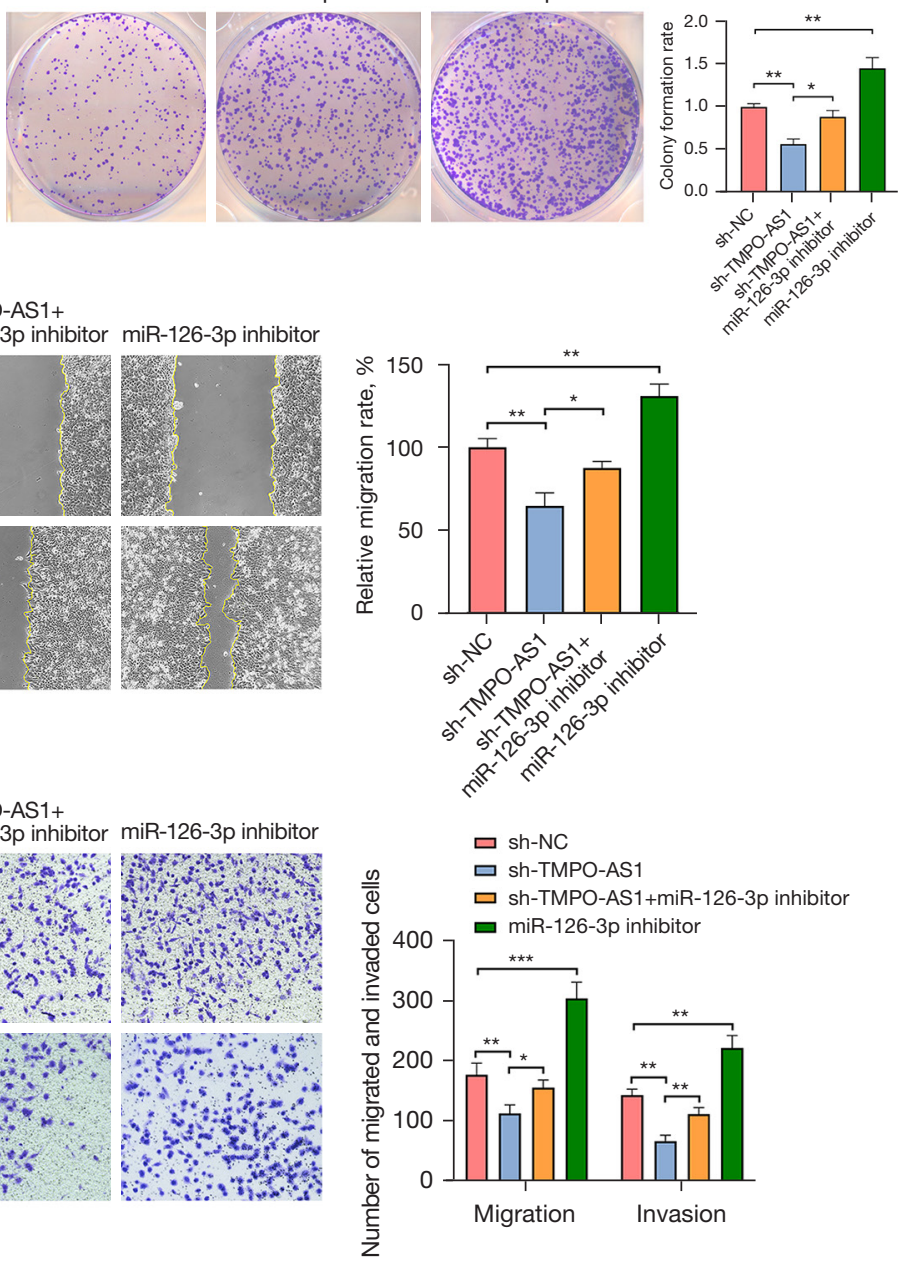

Figure 5 TMPO antisense RNA 1 (TMPO-AS1) promotes hepatocellular carcinoma progression by inhibiting the miR-126-3p/LRP6 axis. (A,B) Cell Counting Kit-8 and colony formation assays showed that decreased miR-126-3p expression recovered the growth-inhibitory role of short-hairpin (sh)-TMPO-AS1 in Huh7 cells. (C,D) Wound healing and transwell assays were conducted to evaluate the ability of Hih7 cells to migrate and invade following cotransfection with sh-TMPO-AS1 and a miR-126-3p inhibitor. Cells were stained with crystal violet. Magnification power 200x. *, $\mathrm{P}<0.05$; **, $\mathrm{P}<0.01 ;{ }^{* * *}, \mathrm{P}<0.001$.

\section{miR-126-3p inbibition abrogates the repressive effect of TMPO-AS1 knockdown}

We next explored how the biological functions of HCC are regulated by TMPO-AS1 in combination with miR126-3p. The results of the CCK8 and clone formation assays indicated Huh7 cell proliferation is decreased following TMPO-AS1 knockdown, as compared with NC. However, cell proliferation can be enhanced by miR-126$3 p$ suppression. Nevertheless, the inhibitory effect of shTMPO-AS1 on HCC growth could be recovered by the repression of miR-126-3p (Figure 5A,5B). Correspondingly, wound healing assays (Figure $5 C$ ) and transwell (Figure 5D) revealed that the reduced ability of sh-TMPO-AS1 to prevent HCC cell migration and invasion could be partially recuperated through miR-126-3p suppression. In general, these results imply that TMPO-AS1 regulates HCC cell metastasis by restraining the miR-126-3p/LRP6 axis.

\section{Discussion}

The recent focus on non-coding RNAs has led to the discovery 
of the biological roles lncRNAs play in distinct human tumors $(20,21)$, providing novel insights into the development of cancer. IncRNA regulates HCC cells in a variety of ways, including DNA binding and chromatin remodeling, RNA interaction, protein interaction and regulation, and small peptide generation (22). Substantial TMPO-AS1 expression has been demonstrated in human cancers, and downregulation of TMPO-AS1 has been suggested to repress proliferation while promoting apoptosis in cancer cells by sequestering cancer-associated miRNAs (23). However, the role of TMPOAS1's in the initiation and progression of liver cancer is not well characterized, with the underlying molecular mechanisms remaining elusive. Our research shows TMPO-AS1 is upregulated in HCC tissues and cell lines. In addition, high expression of TMPO-AS1 can lead to decreased quality of life of patients. Therefore, we suspected that TMPO-AS1 can be used as a potential biomarker for patient risk stratification and local regional metastasis hepatocellular carcinoma.

The induction function of EMT is well-known in all stages of tumor development, such as tumor initiation, tumor distant metastasis, tumor cell migration, intravascular metastasis, and malignant progression (24,25). Some studies have confirmed that EMT is closely related to immunosuppressive cytokines and $\mathrm{T}$ lymphocyte resistance in the development of tumors (26). In addition, there is evidence that EMT is closely associated with broader inflammatory changes in the tumor microenvironment in NSCLC (27). In the last few years, EMT has been shown to play an indispensable role in inducing the invasion and migration of HCC cells (28). It is evident from our results that TMPO-AS1 can trigger the transformation of epithelial traits of HCC cells into mesenchymal characteristics, leading to increased cell invasion and metastasis. In the clinical specimens we studied, patients with high expression of TMPO-AS 1 showed more microvascular invasion. Relatedly, TMPO-AS1 also plays an essential part in promoting the proliferation of HCC cells and may therefore spur the growth of tumors in newly invaded tissues.

While our study confirmed that TMPO-AS1 promotes HCC progression, the underlying mechanism(s) by which TMPO-AS1 regulates HCC pathogenesis remain(s) to be elucidated. Recent research has put forward a novel regulatory mechanism whereby lncRNAs can serve as endogenous competitive 'sponges', which interact with miRNAs to regulate their functions $(29,30)$. We therefore studied the potential downstream signaling pathway employed by TMPO-AS1 to act as competing endogenous
RNA (ceRNA) in the development of HCC. Bioinformatics analysis (starBase 3.0) illuminated a presumptive binding site between TMPO-AS1 and miR-126-3p. Interestingly, existing literature suggests miR-126-3p plays a vital role as a tumor suppressor in liver cancer $(19,31)$. Thus, we postulated that TMPO-AS1 might participate in HCC development by interacting with miR-126-3p. Luciferase reporter and qRTPCR assays indicated that TMPO-AS1 binds to miR-126$3 \mathrm{p}$ and decreases its expression. Meanwhile, LRP6, which has been deemed to be downstream miR-126-3p (19), is a well-established activator of beta-catenin, which can exert metastatic, proliferative, and anti-apoptotic effects in several types of cancers, including HCC. Here, we proved that mir-126-3p inhibitor largely rescued the effects of TMPOAS1 on the biological functions of HCC cells. Meanwhile, TMPO-AS1 competitive binding to miR-126-3p prevented the miRNA-induced inhibition of LRP6 and its downstream beta-catenin expression in vitro. Hence, the new TMPOAS1/miR-126-3p/LRP6 pathway could be a promising prognostic marker and therapeutic target in HCC recurrence and metastasis.

In conclusion, our study found TMPO-AS 1 is a novel oncogene that promotes HCC development by competitively binding to miR-126-3p. Furthermore, TMPO-AS1 induces tumor cell proliferation, metastasis, and EMT by activating the miR-126-3p/LRP6/ $\beta$-catenin pathway. This research elucidated the specific role and mechanism of TMPO-AS 1 in promoting HCC development, which will contribute to the design of new therapeutic strategies for the prevention and treatment of HCC. Moreover, TMPO-AS1 may be a prognostic marker for HCC due to its overexpression.

\section{Acknowledgments}

Funding: This study was supported by the National Natural Science Foundation of China (81703063), and the Chongqing Medical Scientific Research Project (Joint Project of Chongqing Health Commission and Science and Technology Bureau) (2021MSXM056).

\section{Footnote}

Reporting Checklist: The authors have completed the MDAR reporting checklist. Available at https://dx.doi. org/10.21037/atm-21-5593

Data Sharing Statement: Available at https://dx.doi. 
org/10.21037/atm-21-5593

Conflicts of Interest: All authors have completed the ICMJE uniform disclosure form (available at https://dx.doi. org/10.21037/atm-21-5593). The authors have no conflicts of interest to declare.

Ethical Statement: The authors are accountable for all aspects of the work in ensuring that questions related to the accuracy or integrity of any part of the work are appropriately investigated and resolved. All procedures performed in this study involving human participants were in accordance with the Declaration of Helsinki (as revised in 2013). The study was approved by the Medical Ethics Committee of the First Affiliated Hospital of Chongqing Medical University (No. 2021-156) and informed consent was taken from all the patients.

Open Access Statement: This is an Open Access article distributed in accordance with the Creative Commons Attribution-NonCommercial-NoDerivs 4.0 International License (CC BY-NC-ND 4.0), which permits the noncommercial replication and distribution of the article with the strict proviso that no changes or edits are made and the original work is properly cited (including links to both the formal publication through the relevant DOI and the license). See: https://creativecommons.org/licenses/by-nc-nd/4.0/.

\section{References}

1. Bray F, Ferlay J, Soerjomataram I, et al. Global cancer statistics 2018: GLOBOCAN estimates of incidence and mortality worldwide for 36 cancers in 185 countries. CA Cancer J Clin 2018;68:394-424.

2. Faloppi L, Scartozzi M, Maccaroni E, et al. Evolving strategies for the treatment of hepatocellular carcinoma: from clinical-guided to molecularly-tailored therapeutic options. Cancer Treat Rev 2011;37:169-77.

3. Rahbari NN, Mehrabi A, Mollberg NM, et al. Hepatocellular carcinoma: current management and perspectives for the future. Ann Surg 2011;253:453-69.

4. Abbastabar M, Sarfi M, Golestani A, et al. lncRNA involvement in hepatocellular carcinoma metastasis and prognosis. EXCLI J 2018;17:900-13.

5. Hung T, Chang HY. Long noncoding RNA in genome regulation: prospects and mechanisms. RNA Biol 2010;7:582-5.

6. Hu G, Niu F, Humburg BA, et al. Molecular mechanisms of long noncoding RNAs and their role in disease pathogenesis. Oncotarget 2018;9:18648-63.

7. Peng K, Liu R, Yu Y, et al. Identification and validation of cetuximab resistance associated long noncoding RNA biomarkers in metastatic colorectal cancer. Biomed Pharmacother 2018;97:1138-46.

8. Wang X, Sun W, Shen W, et al. Long non-coding RNA DILC regulates liver cancer stem cells via IL-6/STAT3 axis. J Hepatol 2016;64:1283-94.

9. Hu L, Ye H, Huang G, et al. Long noncoding RNA GAS5 suppresses the migration and invasion of hepatocellular carcinoma cells via miR-21. Tumour Biol 2016;37:2691-702.

10. Bi HQ, Li ZH, Zhang H. Long noncoding RNA HAND2AS1 reduced the viability of hepatocellular carcinoma via targeting microRNA-300/SOCS5 axis. Hepatobiliary Pancreat Dis Int 2020;19:567-74.

11. Yang J, Liang B, Hou S. TMPO-AS1 promotes cervical cancer progression by upregulating RAB14 via sponging miR-577. J Gene Med 2019;21:e3125.

12. Huang W, Su X, Yan W, et al. Overexpression of ARregulated IncRNA TMPO-AS1 correlates with tumor progression and poor prognosis in prostate cancer. Prostate 2018;78:1248-61.

13. Sun Y, Han C. Long Non-Coding RNA TMPO-AS1 Promotes Cell Migration and Invasion by Sponging miR140-5p and Inducing SOX4-Mediated EMT in Gastric Cancer. Cancer Manag Res 2020;12:1261-8.

14. He L, Hannon GJ. MicroRNAs: small RNAs with a big role in gene regulation. Nat Rev Genet 2004;5:522-31.

15. Bartel DP. MicroRNAs: genomics, biogenesis, mechanism, and function. Cell 2004;116:281-97.

16. Chiu HS, Llobet-Navas D, Yang X, et al. Cupid: simultaneous reconstruction of microRNA-target and ceRNA networks. Genome Res 2015;25:257-67.

17. Thomson DW, Dinger ME. Endogenous microRNA sponges: evidence and controversy. Nat Rev Genet 2016;17:272-83.

18. Tam C, Wong JH, Tsui SKW, et al. LncRNAs with miRNAs in regulation of gastric, liver, and colorectal cancers: updates in recent years. Appl Microbiol Biotechnol 2019;103:4649-77.

19. Du C, Lv Z, Cao L, et al. MiR-126-3p suppresses tumor metastasis and angiogenesis of hepatocellular carcinoma by targeting LRP6 and PIK3R2. J Transl Med 2014;12:259.

20. Misawa A, Takayama KI, Inoue S. Long non-coding RNAs and prostate cancer. Cancer Sci 2017;108:2107-14.

21. Bhan A, Soleimani M, Mandal SS. Long Noncoding 
RNA and Cancer: A New Paradigm. Cancer Res 2017;77:3965-81.

22. Huang $Z$, Zhou JK, Peng Y, et al. The role of long noncoding RNAs in hepatocellular carcinoma. Mol Cancer 2020;19:77.

23. Qin Z, Zheng X, Fang Y. Long noncoding RNA TMPOAS1 promotes progression of non-small cell lung cancer through regulating its natural antisense transcript TMPO. Biochem Biophys Res Commun 2019;516:486-93.

24. Nieto MA, Huang RY, Jackson RA, et al. EMT: 2016. Cell 2016;166:21-45.

25. De Craene B, Berx G. Regulatory networks defining EMT during cancer initiation and progression. Nat Rev Cancer 2013;13:97-110.

26. Kudo-Saito C, Shirako H, Takeuchi T, et al. Cancer metastasis is accelerated through immunosuppression during Snail-induced EMT of cancer cells. Cancer Cell 2009;15:195-206.

27. Lou Y, Diao L, Cuentas ER, et al. Epithelial-Mesenchymal Transition Is Associated with a Distinct Tumor

Cite this article as: Huang W, Chen Q, Dai J, Zhang Y, Yi Y, Wei X. Long noncoding TMPO antisense RNA 1 promotes hepatocellular carcinoma proliferation and epithelialmesenchymal transition by targeting the microRNA-126-3p/ LRP6/ $\beta$-catenin axis. Ann Transl Med 2021;9(22):1679. doi: 10.21037/atm-21-5593
Microenvironment Including Elevation of Inflammatory Signals and Multiple Immune Checkpoints in Lung Adenocarcinoma. Clin Cancer Res 2016;22:3630-42.

28. Zhang Y, Shi K, Liu H, et al. miR-4458 inhibits the epithelial-mesenchymal transition of hepatocellular carcinoma cells by suppressing the TGF- $\beta$ signaling pathway via targeting TGFBR1. Acta Biochim Biophys Sin (Shanghai) 2020;52:554-62.

29. Wang $Y, X u Z$, Jiang J, et al. Endogenous miRNA sponge lincRNA-RoR regulates Oct4, Nanog, and Sox2 in human embryonic stem cell self-renewal. Dev Cell 2013;25:69-80.

30. Tay Y, Rinn J, Pandolfi PP. The multilayered complexity of ceRNA crosstalk and competition. Nature 2014;505:344-52.

31. Chen H, Miao R, Fan J, et al. Decreased expression of miR-126 correlates with metastatic recurrence of hepatocellular carcinoma. Clin Exp Metastasis 2013;30:651-8.

(English Language Editor: B. Meiser) 